\title{
Corrigendum of Mean Quadratic Variations and Fourier Asymptotics of Self-similar Measures
}

By

Ka-Sing Lau, Pittsburgh, PA, and Jianrong Wang, College Station, TX

On page 109 we defined the set $\Lambda(t)$ as

$$
\Lambda(t)=\left\{J \in \Lambda: \rho t<\rho_{J} \leqslant t\right\},
$$

where $\rho=\min \left\{\rho_{j}: j=1, \ldots, m\right\}, \rho_{J}=\rho_{j_{1}}, \ldots, \rho_{j_{k}}$ and $J=\left(j_{1}, \ldots, j_{k}\right)$ for some $k$. We remarked that for each infinite sequence $\left(j_{1}, j_{2}, \ldots\right)$ with $j_{i}=1, \ldots, m, i=1,2, \ldots$, there is one and only one $k$ such that $\left(j_{1}, \ldots, j_{k}\right) \in \Lambda(t)$.

This is not true in general. There may be more than one such $k$ that

$$
\rho t<\rho_{j_{1}} \ldots \rho_{j_{k}} \leqslant t
$$

for each infinite sequence $\left(j_{1}, j_{2}, \ldots\right)$.

The correct definition of $\Lambda(t)$ is

$$
\begin{aligned}
\Lambda(t)=\left\{J=\left(j_{1}, \ldots, j_{k}\right) \in \Lambda: \rho t\right. & <\rho_{j_{1}} \ldots \rho_{j_{k}} \leqslant t \\
& \left.\rho_{j_{1}} \ldots \rho_{j_{k-1}}>t \text { if } k>1\right\} .
\end{aligned}
$$

This means for each infinite sequence $\left(j_{1}, j_{2}, \ldots\right)$ with $1 \leqslant j_{i} ! \leqslant m$, we curtail the sequence at the least value of $k \geqslant 1$ for which

$$
\rho t<\rho_{j_{1}} \ldots \rho_{j_{k}} \leqslant t
$$

$\Lambda(t)$ is the set of finite sequences obtained in this way.

K. S. LAU

Department of Mathematics

University of Pittsburgh

Pittsburgh, PA 15260

USA
J. WANG

Department of Mathematics Texas A \& M University College Station, TX 77843 USA 